\title{
Phase function encoding of diffractive structures
}

\author{
Andreas Schilling and Hans Peter Herzig
}

\begin{abstract}
We analyzed the direct sampling (DS) method for diffractive lens encoding, using exact electromagnetic diffraction theory. In addition to previously published research [Pure Appl. Opt. 7, 565 (1998)] we present what we believe to be new results for TM polarization. We found that the validity of the scalar-based DS method is even more extended for TM than for TE polarization. Additionally, we fabricated and characterized DS-encoded blazed gratings and found good agreement between the experimental and theoretical diffraction efficiencies. We analyzed quantitatively the influence of the encoding schemes DS and analytic quantization (AQ) on the quality of the focal spot. We also investigated the focal spot sizes (FWHM) and the Strehl ratios of the DS- and the AQ-encoded cylindrical lenses. (C) 2000 Optical Society of America
\end{abstract}

OCIS codes: $\quad 050.0050,050.1380,050.1970$.

\section{Introduction}

Diffractive optical elements can be found in many applications, ${ }^{1-3}$ and they bring additional degrees of freedom to the application design compared with conventional refractive optics. For the design of optical systems, ray-tracing programs offer the possibility to include arbitrary phase functions, which can be realized by diffractive optical elements, to take advantage of these additional freedoms. The designed phase functions are in the general case continuous and have to be quantized into a multilevel staircaselike profile for a fabrication by the multilevel approach. ${ }^{4}$ Direct sampling (DS) ${ }^{5}$ is a straightforward method to obtain directly the encoded phase profile from a continuous phase function, in contrast to encoding methods such as radially symmetric iterative discrete on-axis encoding, introduced by Welch et al., ${ }^{6}$ in which the quantized phase function is obtained by means of optimizing the energy in a signal window for a given optical setup. Since the fabrication technologies are constantly improving, the possible feature sizes that can be reliably fabricated are shrinking. When typical feature sizes are comparable with the wavelength of the incident light, a purely scalar ap-

The authors are with the Institute of Microtechnology, University of Neuchâtel, Rue A.-L. Breguet 2, CH-2000 Neuchâtel, Switzerland. The e-mail address for A. Schilling is Andreas.Schilling@ imt.unine.ch.

Received 30 March 2000; revised manuscript received 7 July 2000.

0003-6935/00/295273-07\$15.00/0

(C) 2000 Optical Society of America proach becomes insufficient or at least questionable. Reference 7 describes a previous study in which we applied exact electromagnetic theory to the encoding problem to determine the limits of validity for the scalar-based encoding method DS. In this paper we present new results for TM polarization and compare the two polarization cases. Since arbitrary phase functions can be treated locally as linear blazed gratings, we did the rigorous analysis of the quantization for ideally blazed surface-relief phase gratings. In addition, we fabricated and characterized test structures that were encoded with the DS method. We found good agreement between the measured efficiencies and the theoretical efficiencies obtained by exact electromagnetic diffraction theory.

The research performed so far on the encoding problem has focused mainly on the diffraction efficiencies of the encoded phase functions. The influence of the encoding method on the quality of the wave front and therefore on the focal spot size or the Strehl ratio has not yet been investigated. We investigated quantitatively the influence of the encoding scheme on the quality of the wave front for an incident plane wave that is focused by a cylindrical lens encoded by DS or analytic quantization (AQ). Hereto we compared the FWHM of the focal spot and the Strehl ratio of the DS- and the AQ-encoded cylindrical lenses with the ideal case. Whereas for AQ the periodicity of the phase function to be encoded is maintained, DS shows a principally different behavior. In the general case DS enlarges the periodicity of the underlying phase function, thus generating additional diffraction orders in between the main or- 


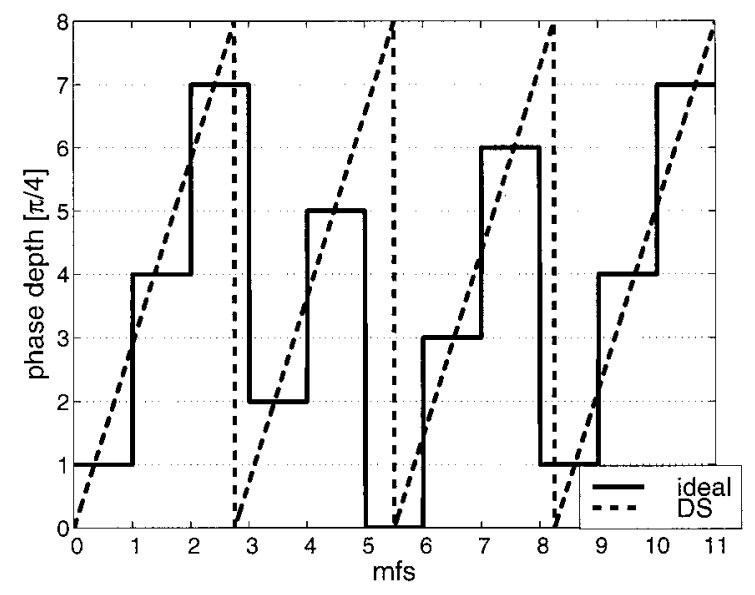

Fig. 1. Dashed line, ideal phase function of a blazed grating $\left(\Lambda_{b}\right)$ MFS $=2.75$ ); solid line, multilevel profile obtained by DS.

ders. We discuss the consequences of this difference between the two encoding schemes.

\section{Theoretical Background}

In this section we introduce the theoretical background used in Section 3. First, we introduce the encoding methods DS and $\mathrm{AQ}$ and explain how the rigorous and the scalar diffraction efficiencies were calculated. Afterward, we show how the fields were propagated for the focal spot analysis in Subsection 3.C.

The great advantage of the encoding method DS is its simplicity and general applicability. For DS coding the complete lens function is sampled with the minimum feature size (MFS), and the phase values are clipped between 0 and $2 \pi$. These phase values are then rounded to the closest available phase level. ${ }^{5}$ The DS encoding method is illustrated in Fig. 1. In $\mathrm{AQ}^{8}$ the phase function is clipped to values between 0 and $2 \pi$; then the transition points for $2 \pi$ phase jumps are determined, and the regions between these transitions are encoded. We determine the number of phase values between two transitions by dividing the distance between the transitions by the MFS and rounding this value to the integer closest to zero. In this paper we address especially high-aperture lenses, since there the influence of the encoding method is most evident. Since nonuniformly distributed phase levels are of advantage only for weak lenses, ${ }^{9}$ we used equally separated phase levels for the encoding.

For the diffraction efficiency calculations for which exact electromagnetic theory was applied (Subsection 3.A) we used a rigorous eigenmode method as described by Turunen ${ }^{10}$ with modifications as published by Moharam et al. ${ }^{11}$ To determine whether the quantized phase profiles obtained with DS are also the optimum solutions, meaning with the highest diffraction efficiencies, we used the same rigorous steepestgradient optimization (RSGO) method as in Ref. 7 The sampling of the lens function was hereby kept constant, 1 MFS. The starting point for the optimi- zation procedure was the phase-level distribution obtained by DS. This phase-level distribution was then optimized with the steepest-gradient algorithm until the optimum solution with the highest diffraction efficiency was found. The scalar diffraction efficiencies, $\eta_{m}$, for the DS- and the AQ-encoded gratings were calculated by a Fourier transform and given by

$$
\eta_{m}=\left|T_{m}\right|^{2}=\left|\frac{1}{d} \int_{0}^{d} t(x) \exp (-i 2 \pi m x / d) \mathrm{d} x\right|^{2},
$$

where $T_{m}$ are the Fourier amplitudes of order $m, t(x)$ the complex amplitude transmission function, $x$ the lateral coordinate, and $d$ the grating period.

For the scalar calculations of the focal plane intensities of the cylindrical lenses in Subsection 3.B we evaluated the Rayleigh-Sommerfeld diffraction integral $^{12}$

$$
\begin{aligned}
u_{2}\left(x, z_{2}\right)= & \int_{-\infty}^{+\infty} u_{1}\left(x^{\prime}, z_{1}\right) \tau\left(x^{\prime}, z_{1}\right) \\
& \times\left(\frac{1}{k r_{12}}-i\right) \frac{z_{12}}{\lambda r_{12}^{2}} \exp \left(i k r_{12}\right) \mathrm{d} x^{\prime},
\end{aligned}
$$

where $u_{1}\left(x^{\prime}, z_{1}\right)$ is the incident wave field, $\tau\left(x^{\prime}, z_{1}\right)$ is the aperture function, $k=2 \pi / \lambda, z_{12}$ is the distance between the two planes located at $z_{1}$ and $z_{2}, r=$ $\sqrt{z_{12}^{2}+\left(x-x^{\prime}\right)^{2}}$, and $\lambda$ is the wavelength.

For all calculations in this study we assumed a linearly polarized plane wave under normal incidence. For the rigorous optimization calculations we assumed a substrate with a refractive index of $n=$ 1.5. The calculations were made for a propagation direction substrate to air, which is the standard case for a diffractive microlens. For the inversed propagation direction no significantly different behavior is expected, as was shown in Ref. 7 We analyzed DSencoded blazed gratings, since any arbitrary lens function can locally be treated as a blazed grating. The local period of a blazed grating, $\Lambda_{b}$, and therefore the $\Lambda_{b} / \lambda$ ratios are smallest in the outermost part of a diffractive high-aperture lens with design wavelength $\lambda$. There are only two or three phase levels possible per $2 \pi$ phase difference, because of the limitations imposed by the MFS. We will call this part of the lens the binary region. Differences between scalar and exact electromagnetic theory are most likely to appear there. We therefore concentrated our rigorous analysis on the binary region.

\section{Results and Discussion}

\section{A. Rigorous Analysis of Diffractive Lens Encoding}

We applied exact electromagnetic diffraction theory to the DS method to determine the limits of validity of the scalar-based method. In addition to the results for TE polarization, which are shown for the sake of completeness and comparison, we show new results for TM polarization and compare the two polarization cases. The rigorous analysis of the DS 

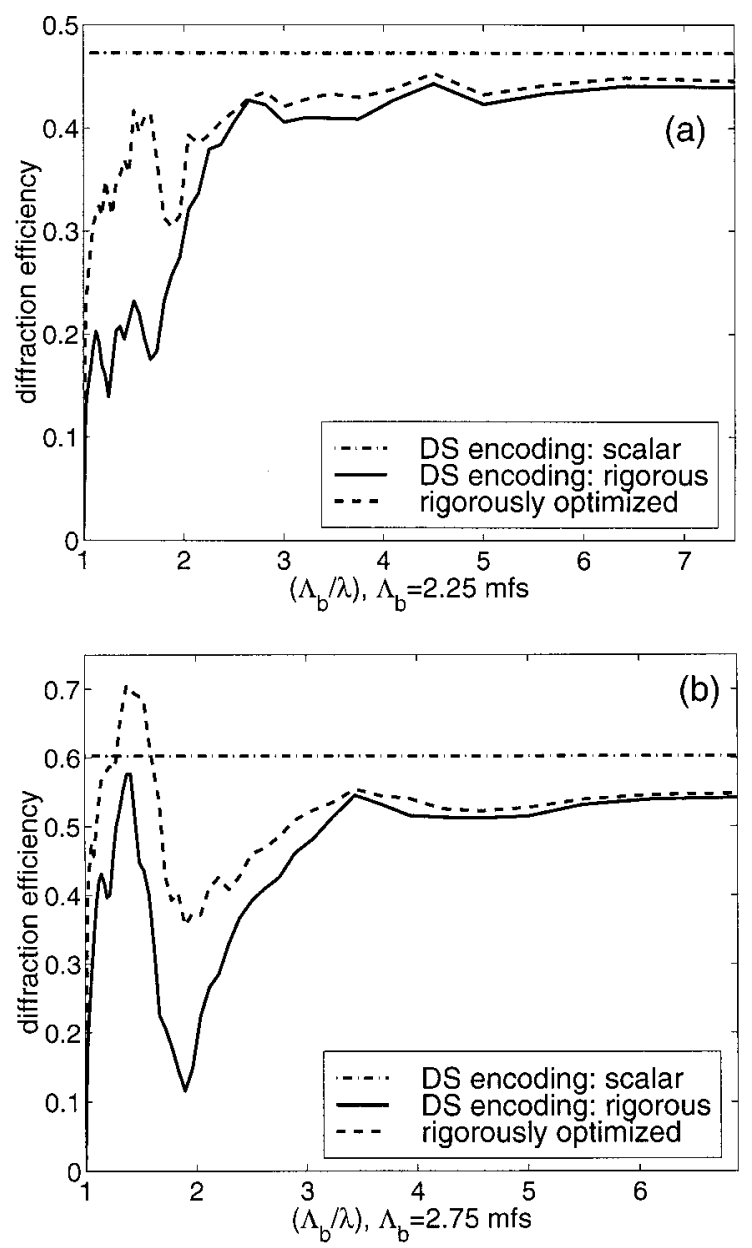

Fig. 2. Calculated diffraction efficiencies (TE polarization) as a function of $\Lambda_{b} / \lambda$ with constant $\Lambda_{b}$ for blazed gratings with (a) $\Lambda_{b} / \mathrm{MFS}=2.25$ and (b) $\Lambda_{b} / \mathrm{MFS}=2.75$. For the phase depth of $2 \pi$, eight equally spaced phase levels were available. Dotteddashed curves, scalar diffraction efficiencies; solid curves, rigorously calculated diffraction efficiencies; dashed curves, rigorous diffraction efficiencies for the phase-level distributions that were obtained by rigorous optimization (RSGO).

blazed grating was made for eight different $\Lambda_{b} / \mathrm{MFS}$ ratios in the binary region, namely, $2,2.2,2.25,2.4$, $2.5,2.6,2.75$, and 2.8, for both polarizations. In Fig. 1 the case $\Lambda_{b} / \mathrm{MFS}=2.75$ is illustrated where the ideal phase function and the corresponding multilevel profile obtained by DS are shown. The results for $\Lambda_{b} /$ MFS values of 2.25 and 2.75 are shown in Fig. 2 for TE polarization and in Fig. 3 for TM polarization. Each graph contains three diffraction efficiency curves: the DS-encoded phase-level distribution, calculated with exact electromagnetic theory (solid curves) and simple scalar theory (dotted-dashed curves), and the RSGO optimized phase-level distribution, calculated with exact electromagnetic theory (dashed curves). The important criterion for the performance of the encoding method is the $\Lambda_{b} / \lambda$ ratio at which the scalar-based encoding method DS produces phase-level distributions that cannot significantly be improved when optimized with exact electromagnetic theory (RSGO). This ra-
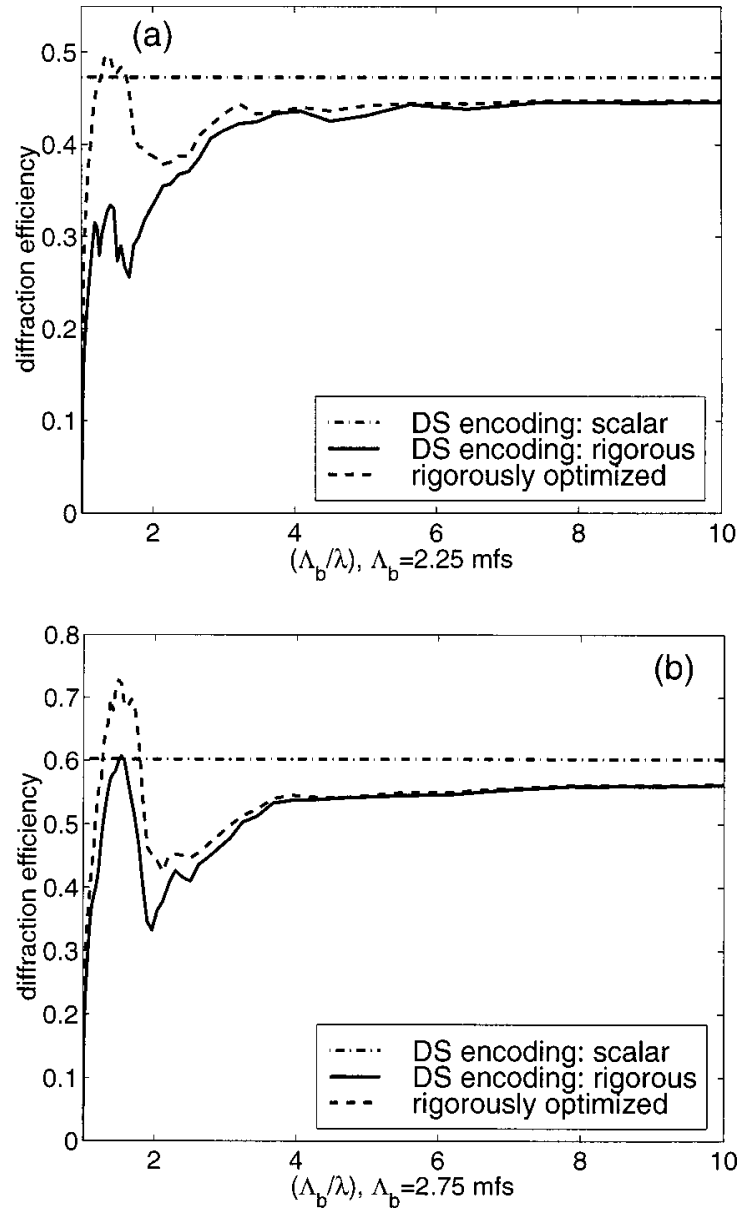

Fig. 3. Calculated diffraction efficiencies (TM polarization) as a function of $\Lambda_{b} / \lambda$ with constant $\Lambda_{b}$ for blazed gratings with (a) $\Lambda_{b} / \mathrm{MFS}=2.25$ and (b) $\Lambda_{b} / \mathrm{MFS}=2.75$. For the phase depth of $2 \pi$, eight equally spaced phase levels were available. Dotteddashed curves, scalar diffraction efficiencies; solid curves, rigorously calculated diffraction efficiencies; dashed curves, rigorous diffraction efficiencies for the phase-level distributions that were obtained by rigorous optimization (RSGO).

tio will be designated $\left(\Lambda_{b} / \lambda\right)_{0}$. We determined $\left(\Lambda_{b} /\right.$ $\lambda)_{0}$ for the eight different $\Lambda_{b} /$ MFS ratios in the binary region for both polarizations. $\left(\Lambda_{b} / \lambda\right)_{0}$ were determined by the condition that for all $\Lambda_{b} / \lambda$ ratios larger than $\left(\Lambda_{b} / \lambda\right)_{0}$ the rigorously optimized solutions show an improvement in diffraction efficiency of less than $3 \%$ over the solutions obtained with DS. The $\left(\Lambda_{b} / \lambda\right)_{0}$ ratios are shown in Table 1 for TE polarization and in Table 2 for TM polarization. The values that were obtained for $\left(\Lambda_{b} / \lambda\right)_{0}$, between 2 and 3.3 for TE polarization, and between 2 and 2.8 for TM polarization, are very small compared with the normal validity range of scalar diffraction theory for grating structures $\left[\left(\Lambda_{b} / \lambda\right) \geqslant 10\right] .^{13}$ The values for TM polarization are hereby even smaller than the values obtained for TE polarization. The results show that simple DS encoding ensures optimum performance in the above sense over a wide $\Lambda_{b} / \lambda$ range for both polarizations, from the scalar regime to deep into the rigorous regime $\left(\Lambda_{b} / \lambda \approx 2-3\right)$, whereas the optimum 
Table 1. $\left(\Lambda_{b} / \lambda\right)_{0}$ Values for Different $\Lambda_{b} /$ MFS Ratios of the DS Blazed Grating in the Binary Region: TE Polarization ${ }^{a}$

\begin{tabular}{ll}
\hline$\Lambda_{\mathrm{b}} / \mathrm{MFS}$ & $\left(\Lambda_{\mathrm{b}} / \lambda\right)_{0}$ \\
\hline 2.0 & 2.0 \\
2.2 & 2.2 \\
2.25 & 2.3 \\
2.4 & 2.8 \\
2.5 & 2.3 \\
2.6 & 2.9 \\
2.75 & 3.3 \\
2.8 & 3.3 \\
\hline
\end{tabular}

${ }^{a}$ Eight equally spaced levels were available for $2 \pi$ phase.

performance is extended even more for TM polarization than for TE polarization. In the regime $\Lambda_{b} / \lambda<$ $\left(\Lambda_{b} / \lambda\right)_{0}$, where the DS-quantized phase profiles have diffraction efficiencies as low as $11 \%$, the rigorously optimized solutions (RSGO) show large improvements of as much as $25 \%$ for TE polarization and as much as $22 \%$ for TM polarization. Therefore the optimization potential is comparable for TE and TM polarization, whereas the validity of DS is more extended for TM polarization than for TE polarization.

B. Fabrication and Characterization of Direct-Sampling-Encoded Blazed Gratings

We fabricated DS-encoded linear blazed gratings with eight phase levels in fused silica with $\Lambda_{b}=2.25$ MFS and 2.75 MFS for MFS of $1,1.5$, and $2 \mu \mathrm{m}$ and characterized the first-order diffraction efficiencies for both polarizations. The gratings were fabricated by a three-step photolithographic process in which each lithographic step is followed by a reactive ionetching step to produce the eight-level surface profile. The fabrication process was carried out at the Centre Suisse d'Electronique et de Microtechnique in Neuchâtel. Figure 4 shows SEM pictures of the fabricated structures with $\Lambda_{b}=2.25 \mathrm{MFS}$ and $\Lambda_{b}=2.75$ MFS for a MFS of $2 \mu \mathrm{m}$. Numbers 0-7 indicate the corresponding eight phase levels. Subsequently we compared the measured diffraction efficiencies with the values obtained by exact electromagnetic diffraction theory. The results are shown in Figs. 5(a) (TE polarization) and 5(b) (TM polarization) for $\Lambda_{b}=2.25$ MFS and in Figs. 6(a) (TE polarization) and 6(b) (TM polarization) for $\Lambda_{b}=2.75$ MFS. We found good

Table 2. $\left(\Lambda_{b} / \lambda\right)_{0}$ Values for Different $\Lambda_{b} /$ MFS Ratios of the DS Blazed Grating in the Binary Region: TE Polarization ${ }^{a}$

\begin{tabular}{ll}
\hline$\Lambda_{\mathrm{b}} / \mathrm{MFS}$ & $\left(\Lambda_{\mathrm{b}} / \lambda\right)_{0}$ \\
\hline 2.0 & 1.85 \\
2.2 & 2.05 \\
2.25 & 2.12 \\
2.4 & 2.08 \\
2.5 & 2.05 \\
2.6 & 2.24 \\
2.75 & 2.54 \\
2.8 & 2.27 \\
\hline
\end{tabular}

${ }^{a}$ Eight equally spaced levels were available for $2 \pi$ phase.
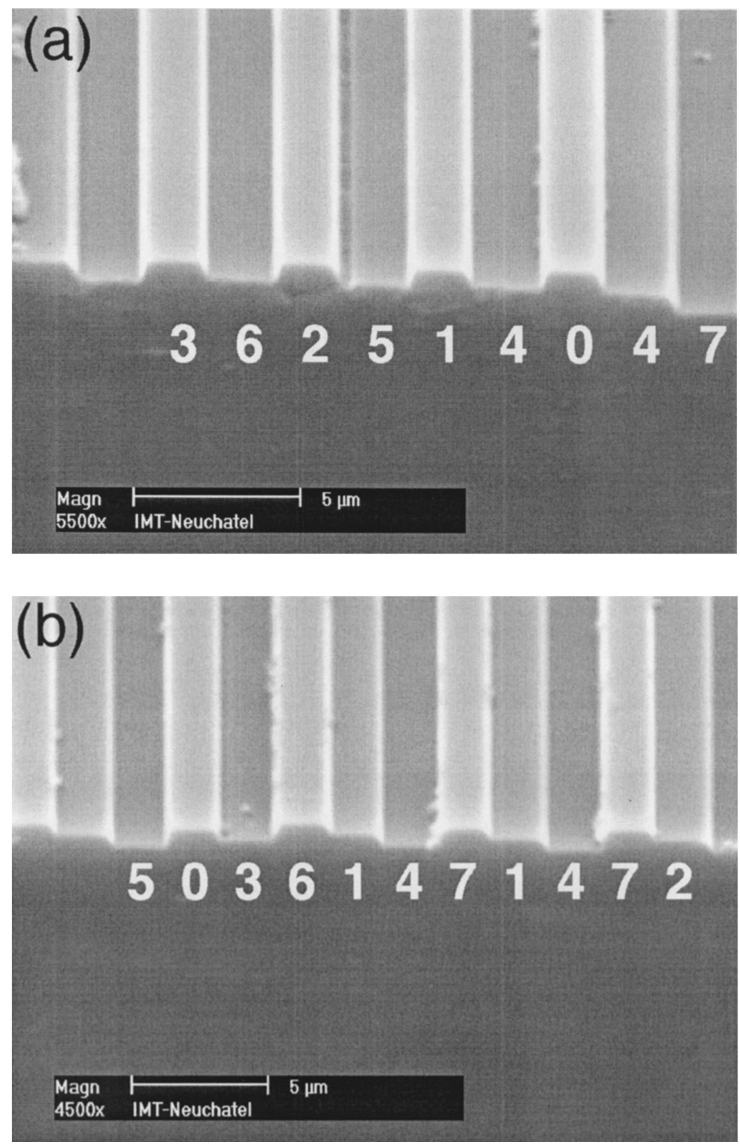

Fig. 4. SEM pictures of the fabricated eight-level DS-encoded blazed gratings in fused silica: (a) for a grating period of 2.25 MFS, (b) for a grating period of 2.75 MFS. Numbers 0-7 indicate the eight different phase levels.

agreement between the experimental and the theoretical values for both polarizations, whereas the measured efficiencies were slightly lower than the theoretical values, which is a result of the alignment errors between the subsequent photolithographic steps during the fabrication process.

\section{Encoding Influence on the Focal Spot}

To investigate the influence of the encoding method on the quality of the focal spot, we calculated the focal spot size (FWHM) and the Strehl ratio as a function of MFS for DS- and AQ-encoded cylindrical lenses as well as for the ideal continuous case. The Strehl ratio for the DS- and the AQ-encoded lenses is defined as the peak intensity in the focal plane divided by the peak intensity of the ideal lens. The focal plane intensities were calculated by evaluation of the Rayleigh-Sommerfeld diffraction integral as given in Eq. (1), which is a standard way of calculating the focal plane intensities of diffractive lenses., ${ }^{5,6}$ The focal plane was defined by the on-axis point with maximum intensity. In Figs. 7(a) and 7(b) the focal spot size and the Strehl ratio, respectively, are shown for cylindrical microlenses with a radius of $r=200$ $\mu \mathrm{m}$, eight phase levels, a wavelength of $0.5 \mu \mathrm{m}$, and 

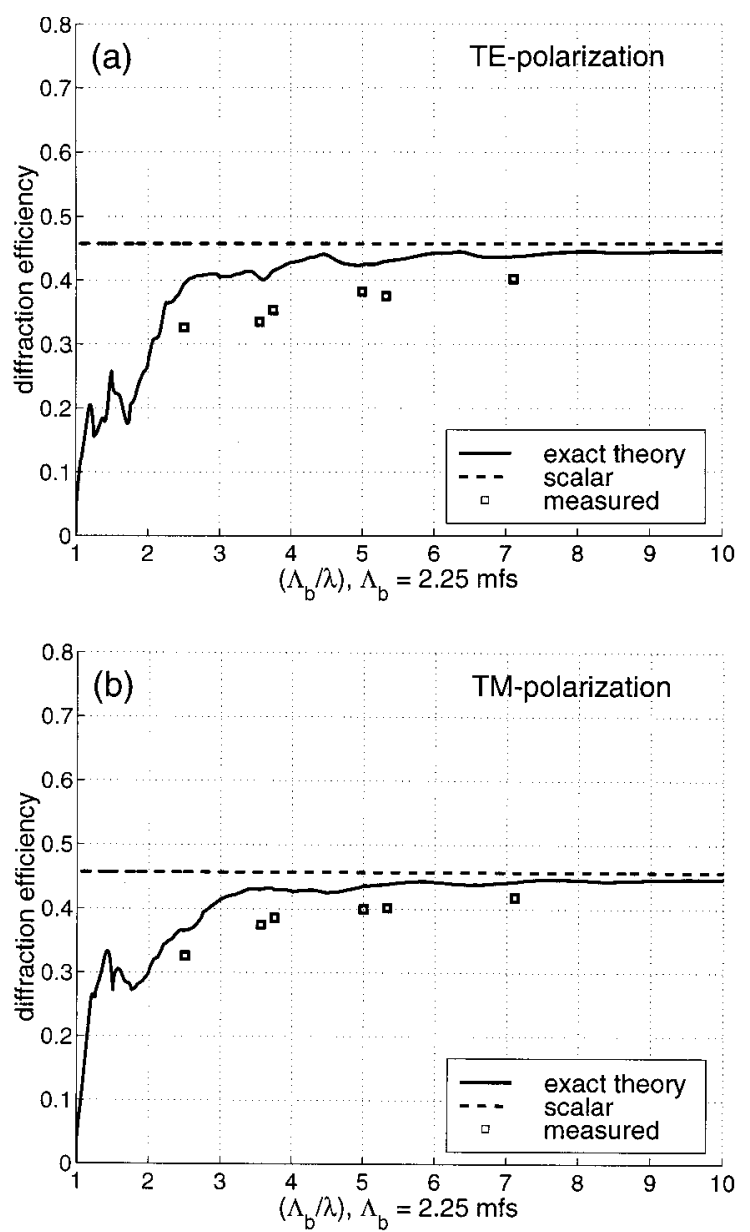

Fig. 5. Experimental and theoretical diffraction efficiencies for (a) TE and (b) TM polarization. The gratings are eight-level DSencoded fused-silica gratings with $\Lambda_{b}=2.25$ MFS.

the minimum focal length. The minimum focal length depends on the MFS, which is determined by the smallest possible grating period of $2 \mathrm{MFS}$ at the edge of the lens. The phase function $\phi$ of the ideal lens is hereby given through

$$
\phi(\rho)=(2 \pi / \lambda)\left[f-\left(f^{2}+\rho^{2}\right)^{1 / 2}\right],
$$

where $f$ is the focal length, $\rho$ the radial coordinate, and $\lambda$ the wavelength. The focal spot sizes for DS, $\mathrm{AQ}$, and the ideal case all show a nearly linear dependence from MFS. The three curves seem to originate from one common point with different slopes. The difference in focal spot size between DS and AQ is quite small compared with the difference between DS and the ideal case. As expected, DS produces smaller focal spots than $\mathrm{AQ}$ for all MFS, and the difference between $\mathrm{DS}, \mathrm{AQ}$, and the ideal case vanishes when the MFS approaches the wavelength, 0.5 $\mu \mathrm{m}$. In our investigation we characterized the focal spot by the FWHM. For other definitions, such the full width at $1 / e^{2}$ intensity level or the width between the first two minima, we expect no significantly different results. The Strehl ratios are practically independent of the MFS for the maximum numerical
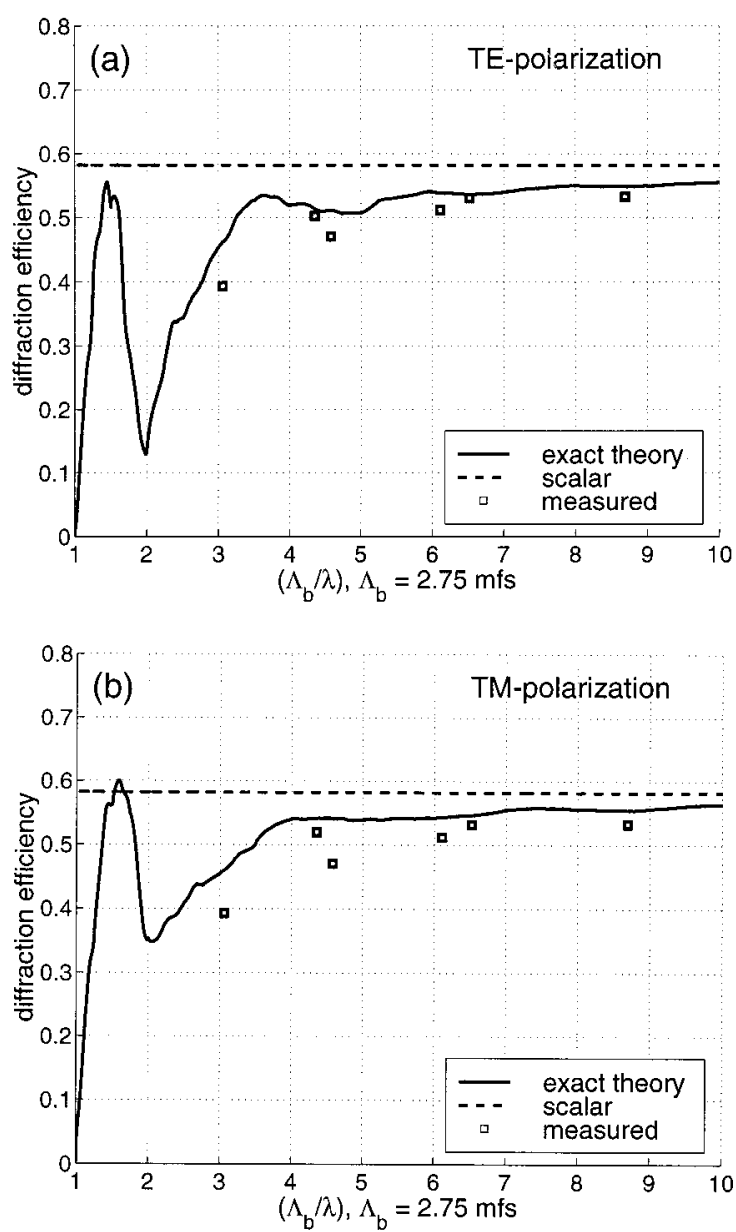

Fig. 6. Experimental and theoretical diffraction efficiencies for (a) TE and (b) TM polarization. The gratings are eight-level DSencoded fused-silica gratings with $\Lambda_{b}=2.75$ MFS.

aperture lenses studied here. The average value for DS is 0.71 and 0.64 for AQ. The formula of Marechal $^{14}$

$$
D_{s}=1-4 \pi^{2}\left(W_{\mathrm{rms}} / \lambda\right)^{2},
$$

relates the Strehl ratio $D_{s}$ with the rms wave-front aberrations, $W_{\text {rms }}$, for a wavelength $\lambda$. Using this formula, we find $W_{\text {rms }}=0.086 \lambda$ for the DS-encoded lens and $W_{\text {rms }}=0.095 \lambda$ for the $\mathrm{AQ}$-encoded lens. Therefore the wave-front aberrations caused by the encoding are $\sim 10 \%$ smaller for DS than for AQ in the case of the investigated high-aperture lenses.

Because of the enlarged period of the DS-encoded blazed gratings, compared with that for the nonencoded blazed phase function, there are additional diffraction orders created in between the main diffraction orders. This principal difference between the two encoding schemes is shown in Figs. 8(a) and 8(b) for DS- and AQ-encoded blazed gratings with $\Lambda_{b}=2.25 \mathrm{MFS}$ and $\Lambda_{b}=2.75 \mathrm{MFS}$, respectively. It can be observed that there are additional diffraction orders generated in between the main diffraction orders but that the absolute magnitude of the intensity level is very small, approximately 1-2 orders of 

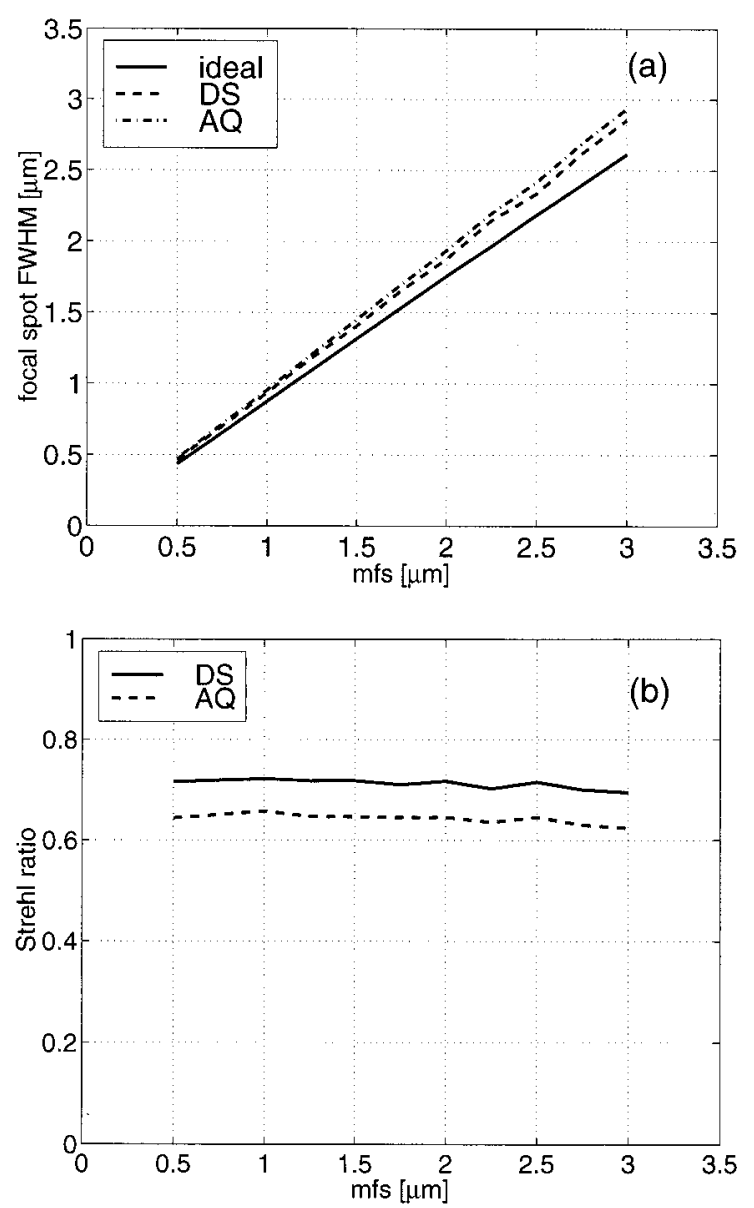

Fig. 7. Focal spot sizes (FWHM) and Strehl ratios for DS- and $\mathrm{AQ}$-encoded cylindrical lenses compared with the ideal case: (a) FWHM as a function of MFS, (b) Strehl ratio as a function of MFS.

magnitude smaller than in the main orders. This is consistent with the results for the focal spot sizes and the Strehl ratios. The difference between the focal spot sizes is quite small for DS and $\mathrm{AQ}$, since the intermediate diffraction orders have a small influence, owing to the small magnitude, whereas the Strehl ratio differs significantly for DS and AQ, because the first-order efficiencies for DS are significantly higher than for AQ.

\section{Conclusions}

DS-encoded blazed gratings have been analyzed with exact electromagnetic diffraction theory. The limits of the scalar-based encoding method DS were determined for both polarizations where we found that the validity of the method clearly exceeds the scalar regime. The DS method produces, under given fabrication constraints, optimum solutions for $\Lambda_{b} / \lambda$ ratios as small as $\sim 3$. The optimum performance of the method is even more extended for TM polarization than for TE polarization. We fabricated and characterized eight-level DS-encoded blazed gratings in fused silica and found good agreement between the experimental and the theoretical diffraction efficiencies. We compared the influence of the encoding
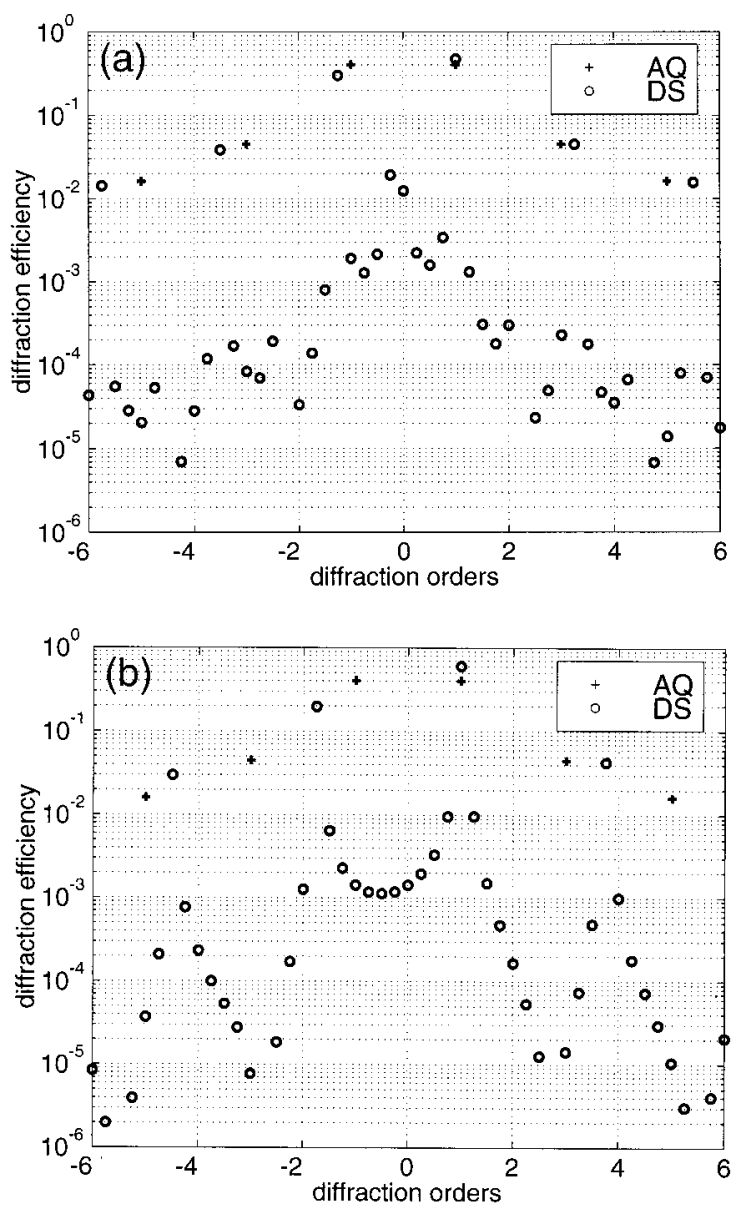

Fig. 8. Efficiencies in the various diffraction orders for DS- and AQ-encoded blazed gratings: (a) for $\Lambda_{b}=2.25 \mathrm{MFS}$, (b) for $\Lambda_{b}=$ 2.75 MFS.

schemes DS and AQ on the quality of the focal spot. Hereto we compared the size (FWHM) of the focal spot and the Strehl ratio of the DS- and the AQencoded cylindrical lenses with the case of the ideal lens.

This research was supported by the Swiss Priority Program Optique II. The authors thank the Centre Suisse d'Electronique et de Microtechnique (CSEM) in Neuchâtel, Switzerland, for the fabrication of the multilevel gratings in fused silica.

\section{References}

1. G. P. Behrmann and J. N. Mait, "Hybrid (Refractive/Diffractive) Optics," in Micro-Optics: Elements, Systems and Applications, H. P. Herzig, ed. (Taylor \& Francis, London, 1997), pp. 259-292.

2. W. Singer, H. P. Herzig, M. Kuittinen, E. Piper, and J. Wangler, "Diffractive beamshaping elements at the fabrication limit," Opt. Eng. 35, 2779-2787 (1996).

3. P. Blattner, H. P. Herzig, K. J. Weible, J. M. Teijido, H. J. Heimbeck, E. Langenbach, and J. Rogers, "Diffractive optics for compact space communication terminals," J. Mod. Opt. 43, 1473-1484 (1996).

4. M. B. Stern, "Binary optics fabrication" in Micro-Optics: $\mathrm{El}$ ements, Systems and Applications, H. P. Herzig, ed. (Taylor \& Francis, London, 1997), pp. 53-85. 
5. M. Kuittinen and H. P. Herzig, "Encoding of efficient diffractive microlenses," Opt. Lett. 20, 2156-2158 (1995).

6. W. H. Welch, J. E. Morris, and M. R. Feldmann, "Iterative discrete on-axis encoding of radially symmetric computergenerated holograms," J. Opt. Soc. Am. A 10, 1729-1738 (1993).

7. A. Schilling, P. Blattner, and H. P. Herzig, "Direct sampling for diffractive microlens encoding from a rigorous point of view," Pure Appl. Opt. 7, 565-574 (1998).

8. G. J. Swanson, Tech. Rep. 854 (MIT Lincoln Laboratory, Lexington, Mass., 1989)

9. U. Levy, N. Cohen, and D. Mendlovic, "Analytic approach for optimal quantization of diffractive optical elements," Appl. Opt. 38, 5527-5532 (1999).
10. J. Turunen, "Diffraction theory of microrelief gratings," in Micro-Optics: Elements, Systems and Applications, H. P. Herzig, ed. (Taylor \& Francis, London, 1997), pp. 31-52.

11. M. G. Moharam, D. A. Pommet, E. B. Grann, and T. K. Gaylord, "Stable implementation of the rigorous-coupled-wave analysis for surface relief gratings: enhanced transmittance matrix approach," J. Opt. Soc. Am. A 12, 1077-1086 (1995).

12. J. D. Gaskill, Linear Systems, Fourier Transforms, and Optics (Wiley, New York, 1978), pp.361-390.

13. D. A. Pommet, M. G. Moharam, and E. B. Grann, "Limits of scalar diffraction theory for diffractive phase elements," J. Opt. Soc. Am. A 11, 1827-1834 (1994).

14. M. Born and E. Wolf, Principles of Optics (Cambridge University Press, Cambridge, 1997), Chap. 9. 